INSTITUT NATIONAL DE RECHERCHE EN INFORMATIQUE ET EN AUTOMATIQUE

\title{
Extending Mobile IP with Adaptive Individual Paging: A Performance Analysis
}

Claude Castelluccia

$$
\mathbf{N}^{\circ} \mathbf{0 2 3 6}
$$

November 1999

THÈME 1

\section{apport \\ technique}





\title{
RIN RIA
}

\section{Extending Mobile IP with Adaptive Individual Paging: A Performance Analysis}

\author{
Claude Castelluccia * \\ Thème 1 - Réseaux et systèmes \\ Projet PLANETE \\ Rapport technique ${ }^{\circ} 0236$ - November 1999 - 23 pages
}

\begin{abstract}
:
This paper proposes to extend Mobile IP with adaptive individual paging. Paging reduces the signaling cost of Mobile IP making it more adapted to wireless cellular IP networks. By reducing the number of location updates to be sent per mobile host, paging also minimizes power consumption of the mobile devices. In the proposed extension, each mobile host computes dynamically its optimal location area size according to its traffic and mobility parameters. The optimal size is the size that provides the best paging versus location update cost tradeoff and, as a result, minimizes the signaling load in the network. We compare, by simulation, the performance of the proposed scheme with that of Mobile IP [11], a paging scheme that uses fixed size location areas and Hierarchical Mobile IP [5]. We show that our extension provides a significant gain compared to Mobile IP and the fixed size paging scheme and performs as well and sometimes better than Hierarchical Mobile IP. We also show that the integration of adaptive individual paging within Hierarchical Mobile IP outperforms all schemes.
\end{abstract}

Key-words: Mobility, Mobile IP, Cellular IP, Location Area Management, IPv6, Internet

(Résumé : tsvp)

*Email : claude.castelluccia@inrialpes.fr

Unité de recherche INRIA Rhône-Alpes

655, avenue de l'Europe, 38330 MONTBONNOT ST MARTIN (France)

Téléphone : 0476615200 - International: +33476615200

Télécopie : 0476615252 - International: +33476615252 


\section{Intégration d'un mécanisme de paging adaptatif et individuel dans Mobile IP}

\section{Résumé :}

Cet article propose un mécanisme de paging adaptatif et individuel pour Mobile IP. Le paging réduit la signalisation générée par Mobile IP et le rend ainsi mieux adapté aux réseaux cellulaires IP. Dans notre proposition, chaque mobile calcule périodiquement la taille optimale de sa zone de localisation en fonction du nombre d'appels entrants et de ses paramètres de mobilité. Les résultats des simulations démontrent l'intêret d'une telle approche.

Mots-clé : Mobilité, Mobile IP, IPv6, Internet 


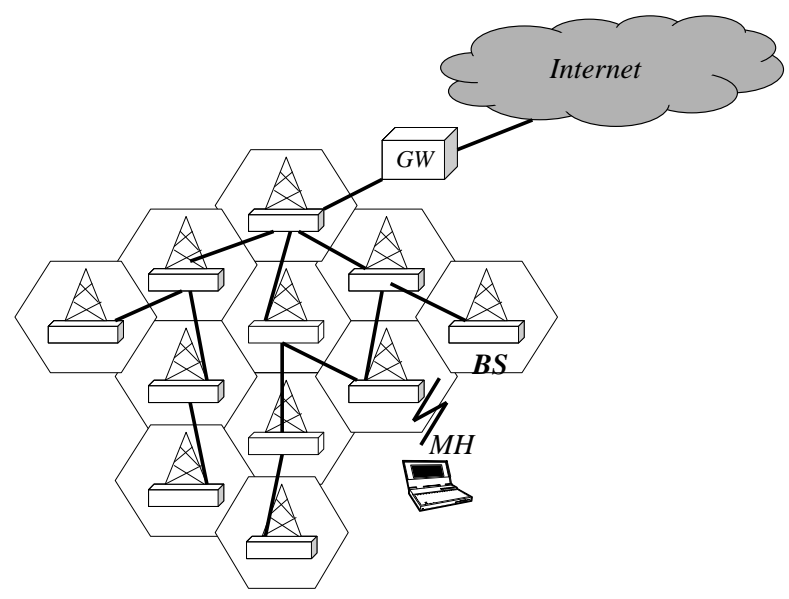

Figure 1: Wireless Cellular Network model

\section{Introduction}

\section{Introduction}

In this paper we consider wireless access to the Internet via cellular IP networks [4, 12]. We envision the next generation of cellular wireless networks as a pure IP-based networks where base stations are IP-routers. A wireless cellular network consists of base stations and routers interconnected by wired links as illustrated in Figure 1. Base stations and routers are both IP-based but base stations have wireless interfaces. Wireless networks are connected to the Internet by gateway routers.

In such networks, mobility should be handled at the IP layer. Different mobility management protocols have been proposed for such networks (HAWAII [12], CellularIP [4],...). Mobile IP [11, 7] is of course a good candidate but needs some extensions to be used efficiently in such environments. In fact, Mobile IP requires that a mobile host sends a binding update (or location update) to its Home Agent whenever its moves from one point-of attachment to another one. These location updates are required even if the mobile host is idle while moving. This signaling cost can become quite significant as the number of mobile hosts increase and the cell sizes get smaller.

Another weakness of mobile IP is its large handoff delay. In Mobile IP the handoff delay is proportional to the round-trip time between the mobile host and its home agent and/or its correspondent hosts (if the route optimization extension [6,7] is used). If the mobile host is far from its home agent, this delay can be significant.

$\mathrm{RT} \mathrm{n}^{\circ} 0236$ 
To overcome this problem, some hierarchical mobility management approaches have been proposed $([3,12,4,5])$. The idea is to define two levels of mobility. The macro level, which corresponds to the mobility between sites or domains, and the micro level, that is the mobility within an administrative domain. All these proposals assume that Mobile IP will be used for the highest level. Different local mobility management protocols are proposed. Although these approaches succeed in solving the handoff delay problem of Mobile IP, they partially solve its high signaling cost problem. In fact, these approaches significantly reduce the load on the backbone but they do nothing about the domain network load.

We propose to use adaptive individual paging for that purpose. Paging is based on the division of the network in several location areas (LA). A mobile host only reports to its home network (or home agent) when it enters a new location area. When the home network needs to contact the mobile host it pages it in its current LA. The mobile host then reports its exact location to its home agent. The size of the location area is very critical for the performance of the paging system. A large location area will generate a high paging cost and will consequently degrade the overall performance. A small location area will not provide the complete benefit of the paging mechanism since it will lead to unnecessary binding updates. The optimal location area size of a host depends on its average speed, the average incoming call rate and its distance to its home network.

This paper evaluates the benefits of such a paging-extended Mobile IP scheme. Section 3 describes the proposed dynamic paging scheme and its integration within Mobile IP. Section 4 explains how the optimal location area size is derived by each mobile host. Section 5 describes the models used in our simulations and analyses the performance results. Section 6 discusses about future work. Section 7 presents the related work. Finally, section 8 concludes the paper.

\section{Extending Mobile IP with individual adaptive paging}

\subsection{Overview}

In cellular systems such as GSM [10], the network tracks mobile users through paging/registration procedures. A paging area consists of a group of base stations where the users are likely to reside. The size of the paging area is fixed for the whole system and depends on the average arrival rate of incoming calls and mobility characteristics of the system users. Paging usually reduces the signaling load on the network since mobile hosts only have to register when they move out of their current location area.

In contrast, Mobile IP tracks mobile users only through registration procedures. As a result, a mobile host has to register with its home agent each time it changes attachment point even if it is idle. We believe that Mobile IP signaling load can be significantly reduced with the use of paging. We therefore extend Mobile IP with some paging capabilities.

However in contrast to existing cellular systems, we define a paging scheme that is individual and adaptive. Since different mobile users may have different incoming call and mobility parameters, a individual scheme in which each mobile host computes its optimal area size should perform better than a system that uses one unique location area size for all users. Similarly, since a mobile host parameters may vary in time, a scheme that adapts a host location area with time is preferable. 
In our proposal, we make the following assumptions:

- Each base station can act as a foreign agent (FA) and a paging agent (PA) for a mobile host. A foreign agent is a router which provides routing services to the mobile node while registered [11]. A paging agent is the base station that is located at the center of a location area and is in charge of the paging process for this area.

- Cells and location areas (LA) have hexagonal shapes. A cell has a radius $R$. A location area is a macro cell with radius $(2 . k-1) . R$ as shown in Figure 2(a). In the rest of this paper, we refer to $k$ as the size of a location area.

- The cost of paging a location area is equal to the cost of sending a signaling message to each of its (micro) cells via some broadcasting mechanisms. The number of cells in a LA of size $k$ is equal to:

$$
s(k)=1+6 \cdot \sum_{i=1}^{k-1} i=1+3 \cdot k \cdot(k-1)
$$

The cost of paging is therefore equal to (see Figure 2(b)):

$$
\operatorname{Paging}_{\text {cost }}(k)=(s(k)-1) \cdot C_{p}=3 \cdot k \cdot(k-1) \cdot C_{p}
$$

where $C_{p}$ is the paging cost in a cell (bytes/paging/cell)

- The cost of a location update is proportional to the geographical distance between the mobile host and the entity it is updating. For example, the cost of a location update from a mobile host (MH) to its home agent (HA) is $d_{M H, H A} \cdot C_{u}{ }^{1}$ where $d_{M H, H A}$ is the geographical distance between the mobile host and its home agent and $C_{u}$ is the location update cost in a cell (bytes/paging/cell).

\subsection{Protocol description}

The operations of our paging-extended Mobile IP protocol are the following:

\section{Mobile host registration}

- When a mobile host detects that it has moved, it registers to a new foreign agent. It then computes its optimal location area size ${ }^{2}, k_{\text {opt }, \text { new }}$ as explained in section 4 .

- the mobile host then decides to change of location area if:

\footnotetext{
${ }^{1}$ This cost has actually to be multiplied by two because location updates to the home agent need to be acknowledged.

${ }^{2}$ The optimal location area size is the size that minimizes the signaling load on the network.
} 


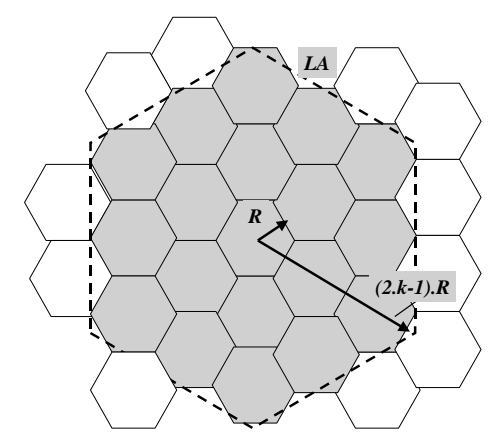

(a) Location Area Model ( $\mathrm{k}=3 ; \mathrm{R}=5)$

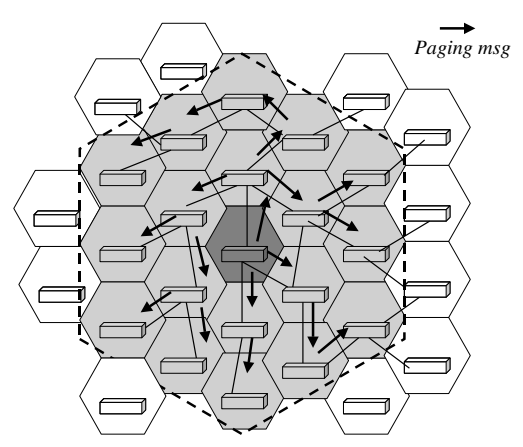

(b) Paging Process

Figure 2: Location Area Model

- its has moved out of its current location area, i.e. if

$$
d_{M H, P A} \geq k_{o p t}
$$

where $d_{M H, P A}$ is the geographical distance between the mobile host and its PA and $k_{o p t}$ is the location area size currently used, or

- if the cost of sending a binding update is lower than the cost that a paging with an outdated location area size generates, i.e., if:

$\left(\operatorname{Paging}_{\text {cost }}\left(k_{\text {opt }, \text { new }}\right)-\right.$ Paging $\left._{\text {cost }}\left(k_{\text {opt }}\right)\right) . P r($ time_between_2_calls $\geq t) \leq d_{M H, H A} . C_{u}$

where $t$ is the elapsed time since the last incoming call of the mobile host.

Since we make the assumption that the incoming calls follow a Poisson process, we have:

$$
\operatorname{Pr}(\text { time_between_2_calls } \geq t)=e^{-R . t}
$$

where $R_{I C}$ is the average number of incoming calls per unit of time.

When a mobile host changes of location area, its current Foreign agent (FA) becomes its Paging Agent. The mobile then sends a binding update to its home agent and registers with its new PA its optimal location area size, $k_{\text {opt, new }}$ (Figure 3(a)).

Assigning the current foreign agent as the paging agent provides a hysteresis such that a mobile host that leaves a LA and creates a new LA, will not immediately return to the previous LA (and re-update its home network) if it crosses back into its previous point-of attachment. This 
reduces the generated signaling load. Another benefit of this scheme is that the update traffic is proportionally distributed in the network. In contrast, update traffic is concentrated on the border of the LAs with fixed LAs.

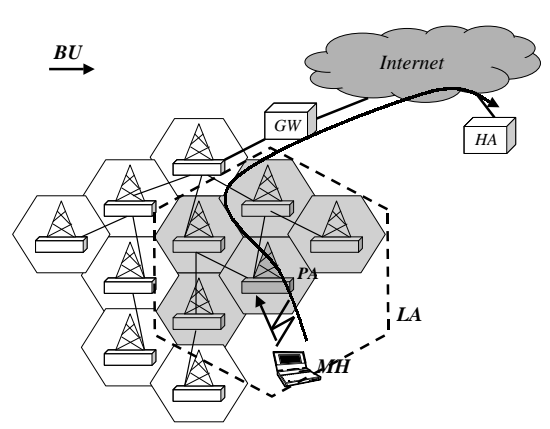

(a) step1: the MH defi nes its LA size and registers with its HA and its PA

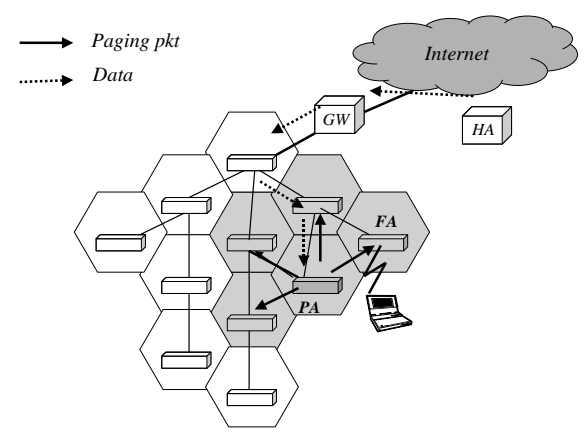

(b) step2: The HA forwards packets to the PA which pages the $\mathrm{MH}$

Figure 3: Paging in Mobile IP

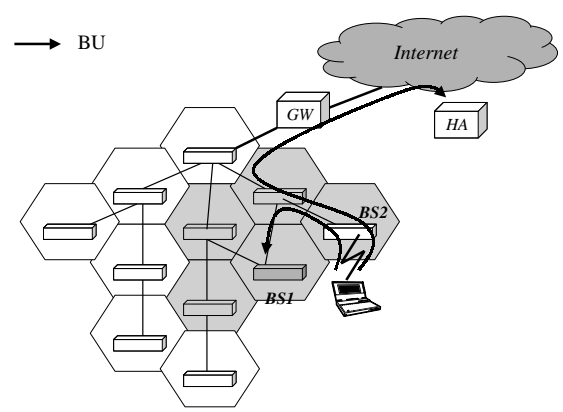

(a) Step3: the MH responds to a paging request by registering its current location to its HA and PA

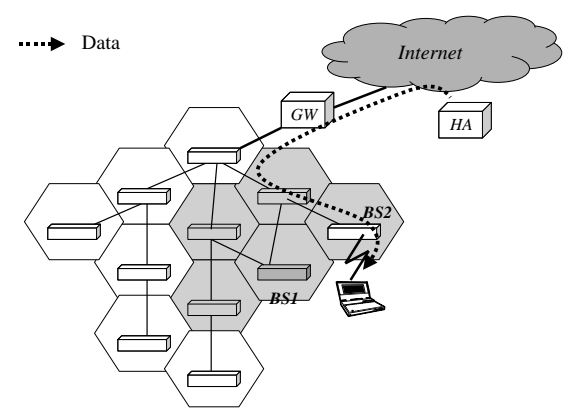

(b) Step4: the HA forwards the packets directly to the MH

Figure 4: Paging in Mobile IP

$\mathrm{RT} \mathrm{n}^{\circ} 0236$ 


\section{Packet delivery}

- When the home agent receives packets for the mobile host, it forwards them to its registered FA (which is actually its PA).

- Upon reception of these packets, the PA buffers them and broadcasts a paging request for the mobile host within the MH's location area size (Figure 3(b)).

- Upon reception of this paging request, the mobile host computes its optimal location area size, $k_{o p t}$ and registers it with its current FA address, which becomes its current PA ${ }^{3}$. It also sends a location update with its current FA to its HA and its previous PA (Figure 4(a)).

- The previous PA and the HA then forward the packets to the MH's current FA (Figure 4(b)).

Note that a buffer management protocol such as the one presented in [8] could be used to minimize loss of data that may happen during the paging process.

\section{Location area optimal size computation}

In this section, we define the signaling cost resulting from the location updates and paging messages. We then use this signaling cost function to derive the optimal paging area size per mobile host.

\subsection{Signaling Cost Function}

The signaling cost function presented in this section is derived from [14]. However [14] only considers the cost on the radio channel. We take into account the signaling cost on the whole network (i.e. the wired and wireless networks). In addition, [14] assumes square-shaped cells and LA. We use, instead, hexagonal shaped cells and location areas. We define the following parameters:

- $R_{I C}$ - the call arrival rate (calls/sec)

- $u_{k}$ - the location update rate per mobile host with the location area size of $k$

- $C_{p}$ - the paging cost in a cell for a call (bytes/paging/cell)

- $C_{u}$ - the location update cost per hop (bytes/update)

- $d_{M H, H A}$ - the distance between a mobile host and its home agent (hops)

- $d_{M H, P A}$ - the distance between a mobile host and the location area FA (hops)

As described in section 3, a paging results in the broadcast of a paging request in the location area followed by a binding update from the mobile host to its home agent and its PA. Note that the binding update to the home agent needs to be acknowledged. The signaling cost per mobile host and per unit time is defined as follows:

\footnotetext{
${ }^{3}$ The PA then adds an entry in its cache that specifi es the location area size associated with the mobile host.
} 


$$
\begin{aligned}
& \operatorname{Cost}\left(k, R_{I C}, u_{k}, d_{M H, P A}, d_{M H, H A}\right)= \\
& R_{I C} \cdot P a g i n g_{\operatorname{cost}}(k) \cdot C_{p}+R_{I C} \cdot\left(d_{M H, P A}+2 . d_{M H, H A}\right) \cdot C_{u}+2 \cdot u_{k} \cdot d_{M H, H A} \cdot C_{u}
\end{aligned}
$$

The first component of this equation represents the paging cost that occurs at each incoming call. The second component is the cost of updating the PA and the home agent after a paging request. The last component corresponds to the cost of updating the home agent when the mobile host moves into a new location area.

For simplicity for assume that $C_{u}=C_{p}=C$. We also make use of the result that for a random movement[14]: $u_{k}=u_{1} / k$.

As a result, we get:

$$
\begin{aligned}
& \operatorname{Cost}\left(k, R_{I C}, u_{k}, d_{M H, P A}, d_{M H, H A}\right)= \\
& \left.3 . k \cdot(k-1)+d_{M H, P A}+2 . d_{M H, H A}\right) \cdot R_{I C} \cdot C+2 \cdot u_{1} \cdot d_{M H, H A} \cdot C / k
\end{aligned}
$$

\subsection{Optimal Location Area Size Derivation}

The optimal location area size for a mobile host, $k_{\text {opt }}$ is defined as the value of $k$ that minimizes the cost function defined by equation 7 .

The first order derivative (equation 8) with respect to $k$ of $\operatorname{Cost}(k)$ shows that $\operatorname{Cost}(k)$ has only one minimum. This minimum is at the intersection of the curve defined by $y=(6 . k-3) \cdot R_{I C} . C$ and the curve defined by $y=2 \cdot u_{1} \cdot d_{M H, H A} \cdot C / k^{2}$.

Furthermore, the second order derivative of $\operatorname{Cost}(k)$ with respect to $k$ (equation 9) is always positive. As a result, $d \operatorname{Cost}(k) / d k$ is a monotonously increasing function of $k$.

$$
\begin{gathered}
d \operatorname{Cost}\left(k, u_{k}, d_{M H, P A}, d_{M H, H A}\right) / d k=(6 \cdot k-3) \cdot R_{I C} \cdot C-2 \cdot u_{1} \cdot d_{M H, H A} \cdot C / k^{2} \\
d \operatorname{Cost}^{2}\left(k, u_{k}, d_{M H, P A}, d_{M H, H A}\right) / d^{2} k=6 \cdot R_{I C} \cdot C+4 \cdot u_{1} \cdot d_{M H, H A} \cdot C / k^{3}
\end{gathered}
$$

Therefore, if we define $\delta()$, the cost difference function between the system with a location area of size $k$ and the system with a location area of size $k-1$ :

$$
\delta\left(k, R_{I C}, C, d_{M H, H A}\right)=3 \cdot(k-1)^{2} \cdot k \cdot R_{I C}-u_{1} \cdot d_{M H, H A} / k \cdot(k-1)
$$

$k_{\text {opt }}$ is defined as follows:

$$
k_{o p t}= \begin{cases}1 & \text { if } \delta\left(2, R_{I C}, u_{1}, d_{M H, H A}\right)>0 \\ \max \left(k: 3 . k(k-1)^{2} \leq u_{1} \cdot d_{M H, H A} / R_{I C}\right) & \text { otherwise }\end{cases}
$$

This result shows that the optimal $k, k_{\text {opt }}$, depends on the mobile host speed, call arrival rate and distance to its home agent.

The following section evaluates the gain that can be achieved via simulation. 


\section{Performance Analysis}

In this section, we evaluate by simulation the performance of the proposed paging-extended Mobile IP proposal. We actually consider four paging schemes for comparison purposes. The first one, $m c p f^{4}$, is a paging-extended Mobile IP scheme that uses fixed location area size. The three others are paging-extended Mobile IP schemes that use individual adaptive location areas but differ in their location area update decision algorithm. With $m c p$, a mobile host changes of location area as soon as its optimal location area size changes. With $m c p 1$, a mobile host changes of location area only when it moves out of its current LA. With mcp_opt, a mobile host changes of LA only if the cost of sending a binding update to its home agent is lower than the cost that a paging with an outdated location area size might generate (this is the scheme used in our proposal).

\subsection{Simulation Model}

In our simulations, we only considered the paging and location update costs. We did not consider the periodic binding updates that a mobile host sends to its home agent or FA to refresh their cache. We developed our own simulation tool to get a better control on it. The network, mobility and incoming call models that we used were adapted from [2].

\section{Network model}

As mentioned previously, we consider a cellular network as an uniform grid of hexagonal cells as illustrated in Fig. 2. A cell has a radius of $R$. $R$ was set to $1 / 2$ in our simulations. The base stations regularly broadcast an $(x, y)$ coordinate of their location. This information is used by the mobile hosts to compute the distance to their home agent or to their PA. Any base station can act as a PA for any mobile host. A location area is defined as a hexagonal area whose size, $k$, is defined by the mobile host.

\section{Host mobility model}

A host mobility consists of micro and macro movements. At the micro level, the user is moving around the vicinity of a fixed location (such as a home, work place,...). We model this micro mobility by a modified random-walk. In the regular random-walk model, a host takes a step of 1 to the West, to the East, to the North or to the South at each time unit. The direction of the move is random. In our modified model, a host only takes a step only every $N$ time unit and stays stationary otherwise. The time between two micro movements follows a Poisson process.

The host occasionally makes large moves (macro moves), e.g. via train or car. The moves are radially symmetric and exponentially distributed, i.e.,

$$
\operatorname{Prob}(\text { size_of_a_big_move } \leq d)=1-e^{-B S t e p . d}
$$

where BStep is the average size of a big move.

\footnotetext{
${ }^{4} m c p$ stands for mobile centered paging. $m c p f$ is a $m c p$ scheme that uses a fi xed location area size.
} 
This big moves occur as a Poisson process with arrival rate $B M$ i.e.

$$
\operatorname{Prob}\left(a \_B i g M o v e \_o c c u r s \_i n_{-} \text {time_interval_T }\right)=1-e^{-B M . T}
$$

Big moves are linear and last one unit of time (like a small move). During a big move, a mobile host gets connected to all the base stations it meets on its way.

In our simulation, the network is modeled as a grid of based station. If the mobile host reaches a border of this grid, it rebounds in the opposite direction.

Figure 5 displays two examples of host mobility. In these two examples, the starting point was set to $(250,250)$ (this is actually the position of the home agent), the number of big moves $(B M)$ was set to 3 and its average size (BStep) to 200. Figure 5(a) represents a "slow-moving" host ( $N$ was set to 4). The mobile host only moves once every 5 time units during micro mobility. Figure 5(b) represents a "fast-moving" host ( $N$ was set to 0$)$. It is moving at each time unit.

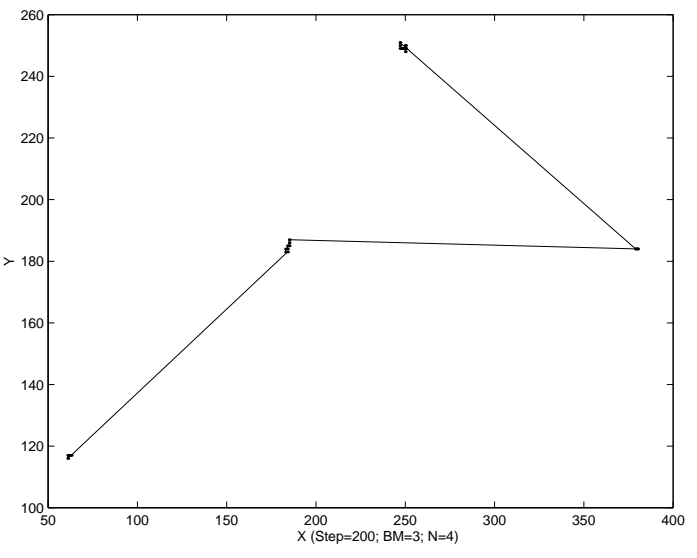

(a) Slow moving host

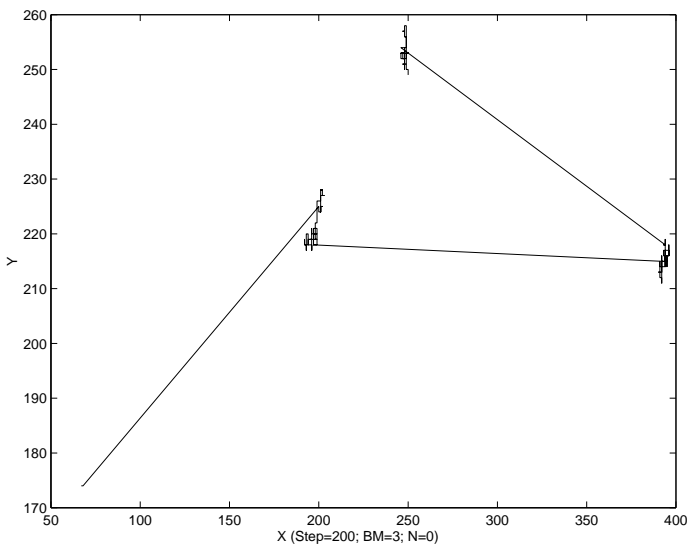

(b) Fast moving host

Figure 5: User mobility model

\section{Incoming call model}

A mobile user receives incoming calls as a Poisson process with rates $a$, i.e.,

$$
\operatorname{Prob}\left(a_{-} c a l l_{\_} o c c u r s \_i n_{-} t i m e \_i n t e r v a l \_t\right)=1-e^{-R_{I C} \cdot t}
$$

We also assume that two successive calls never overlap. In order words, a call always terminate before the beginning of the following one. As a result, the location area pages for the mobile host at each incoming call ${ }^{5}$.

\footnotetext{
${ }^{5}$ We do not consider caching.
} 


\section{Simulation description}

The simulations were executed over a period of $T$ time units ( $T$ was set to 240). A each time unit, the mobile host either stays stationary, makes a micro move (1 step) or makes a big move (BSTEP steps). We then measured the signaling load of different schemes and computed their gain with respect to Mobile IP. The gain of a scheme $X$, is defined as follows:

$$
\operatorname{Gain}_{X}=\operatorname{load}_{X} / \operatorname{load}_{M I P}
$$

where $l o a d_{X}$ and $\operatorname{load}_{M I P}$ are the signaling loads generated respectively by scheme $X$ and Mobile IP during $T$ time units.

The schemes that were considered are: the three variants of dynamic paging ( $m c p, m c p 1$ and $m c p_{-}$opt) and a scheme that uses a fixed location area size for all mobile hosts (different sizes were used). We performed several simulations in which we varied the number of big moves $(B M)$, the average size of a big move (BStep), the idle time period per micro move $(N)$ and the average number of incoming calls per period $\mathrm{T}\left(R_{I C}\right)$. For each simulation configuration (i.e. for each $B M$, $B S t e p, N$ and $R_{I C}$ ), ten different mobility patterns were generated and the results were averaged out.

The results and the analysis of the different simulations are presented in the following section.

\subsection{Analysis}

In the first set of simulations, we fixed $B M$ to $3, B S t e p$ to $200, N$ to 4 and vary $R_{I C}$ from 3 to 33 incoming calls per $T$. The signaling gain of the different schemes are presented in Figure 6(a). For comparison purpose, we displayed in figure 6(b) the gains achieved with $N=0$. The behaviors of the gains of these two figures look very similar. However larger gains are obtained with $N=0$. In fact, in this case most of the movements are local. Paging is then very efficient.

In the rest of this section, we will only consider the case $N=4$.

\section{Dynamic Paging Performance}

These results presented in Figure 6(a) show that mcp_opt outperforms all the other schemes significantly. The achieved gain of this scheme varies from 17 to 8 compared to Mobile IP and is more than two times larger than a scheme using a fixed location area size ( $m c p f 20, m c p f 40$ and $m c p f 60^{6}$ ). This figure shows that the gains of the schemes that use a fixed location area decrease as the number of incoming calls increases. This can be explained by the fact that the number of paging (and therefore of the paging cost) increases linearly as the number of incoming calls increases whereas the number of location updates stay more or less constant (see Figure 8). In contrast, $m c p 1$ gains increase with $R_{I C}$. This is because with $m c p 1$, a mobile host has to wait until the next location update before informing its home agent of its new location area. This leads to a higher paging cost since an outdated location area size is used between the time the mobile computes a new location area size, $t_{1}$ and the time it informs its home agent, $t_{2}$. However, as the number of incoming calls

\footnotetext{
${ }^{6}$ In $m c p f 20, m c p f 40$ and $m c p f 60$ the LA size, i.e. $k$, was respectively set to 20, 40 and 60.
} 


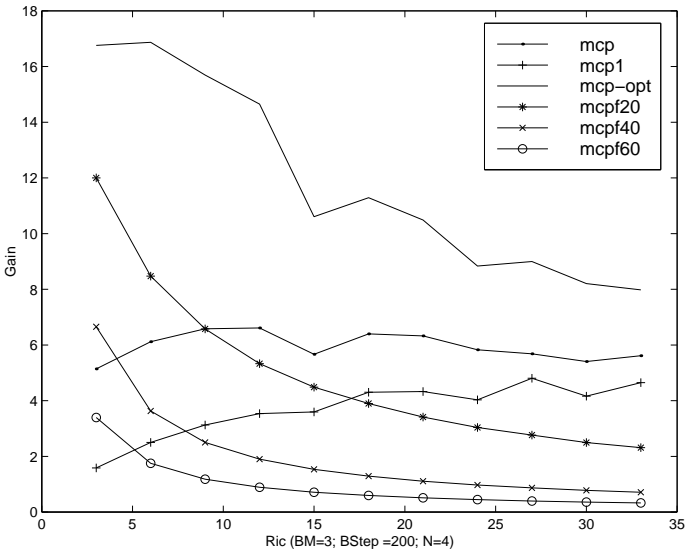

(a) $\mathrm{N}=4$

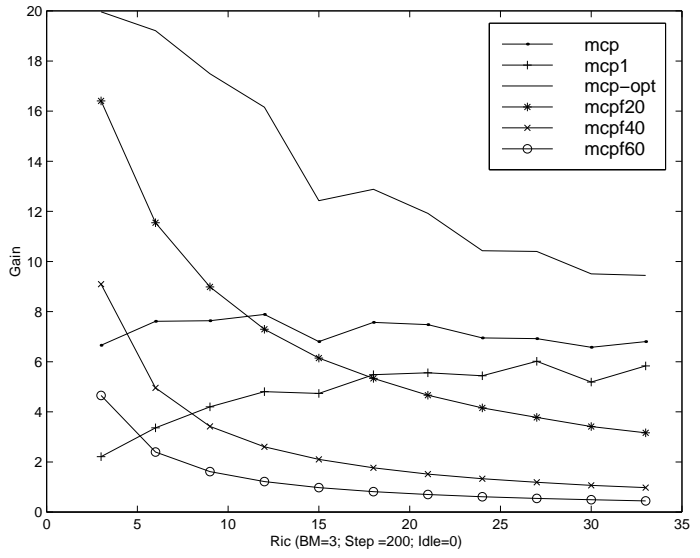

(b) $\mathrm{N}=0$

Figure 6: Signaling gain

increases, the time period $t_{2}-t_{1}$ decreases as a side effect of the location area average size getting smaller (see Figure 7).

$m c p$ gain stays constant as $R_{I C}$ increases. In fact as shown on Figure 8, the location update cost decreases whereas the paging cost increases. These two costs average out to a constant value independent of $R_{I C}$. It is noteworthy that $m c p$ performs better than $m c p 1$. In fact the extra cost of sending a location update immediately after computing a new location area size is smaller than the extra paging cost induced by an outdated location area size.

$m c p_{\text {oopt }}$ gain decreases as $R_{I C}$ increases. As shown in Figure 8, its location update cost is much lower than the $m c p$ one and a bit larger than $m c p 1$. However, $m c p \_o p t$ paging cost is optimal since it is identical to $m c p 1$ paging cost. mcp_opt seems to take the best of $m c p$ and $m c p 1$. It is as adaptive as $m c p 1$ without suffering the extra cost in location update of $m c p$.

Figure 7 displays $m c p \_o p t$ 's average location area size and standard deviation as a function of $R_{I C}$. This figure shows that the average location area size decreases from 60 to 30 as $R_{I C}$ increases from 3 to 33. This result was actually expected. In fact, for a given $d_{M H, H A}$ and $u_{1}$, the largest $k$ that satisfies equation 11 decreases as $R_{I C}$ increases. It also appears that the standard deviation is quite large. This is because the location area size varies quite a lot as the mobile host moves (see figure 9). This result confirms our statement that using a fixed location area size (even per mobile host) can not lead to an optimal solution. An adaptive scheme is better.

Figure 9 displays the instantaneous location area size for $m c p, m c p 1$ and $m c p \_o p t$. The $\mathrm{x}$-axis represents the number of events since the beginning of the mobile host's move. An event is either 


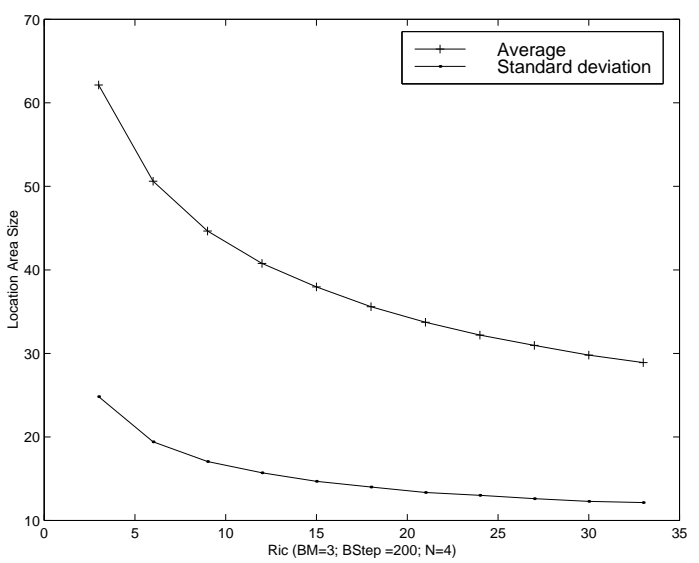

Figure 7: Location Area Size

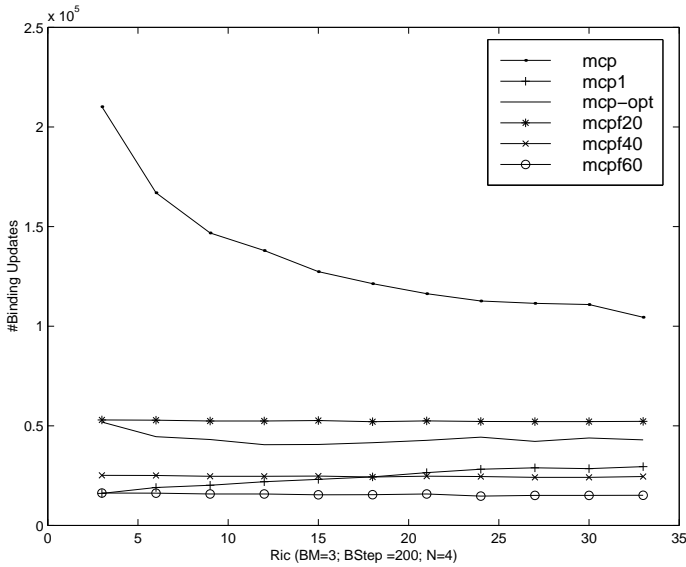

(a) Location Update Cost

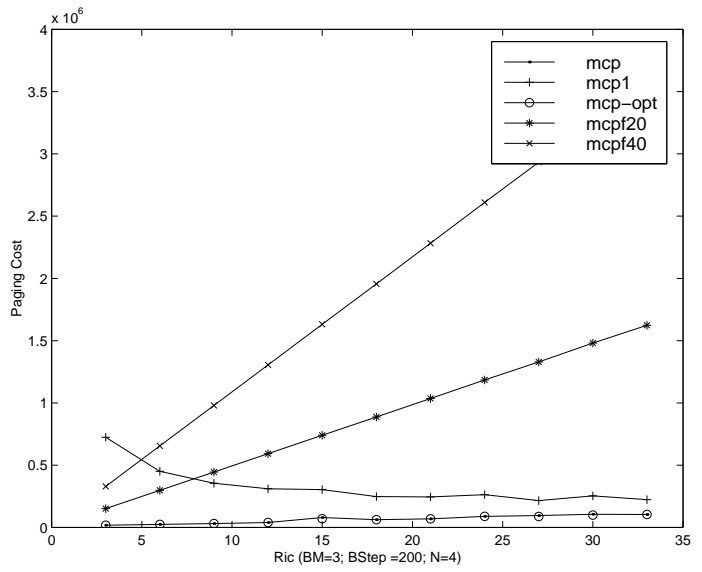

(b) Paging Cost

Figure 8: Signaling cost (BU + Paging)

a small move or an incoming call. A big move generates a burst of events. Figure 9(a) shows that the location area size fluctuates a lot. It starts with a small value (when the mobile host is at home) and then increases progressively until 65 . This increase results from a big move: $d_{M H, H A}$ increases incrementally (see figure 5 - the HA is located at point $(250,250)$ ) and the mobile host speed (i.e., $u_{1}$ ) 


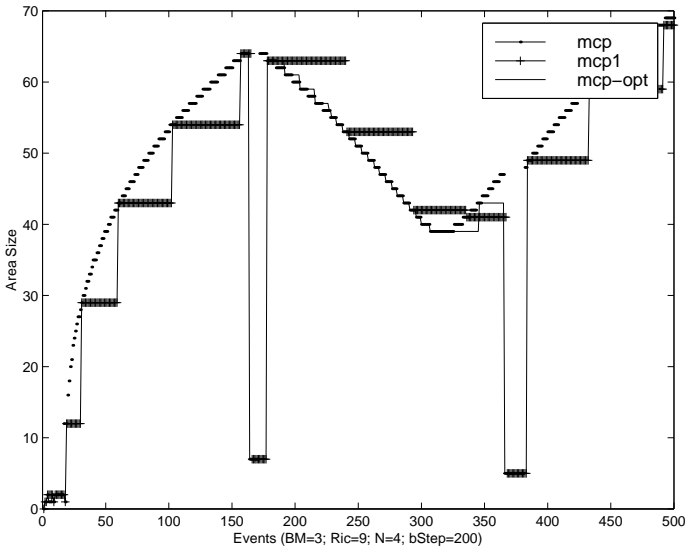

(a)

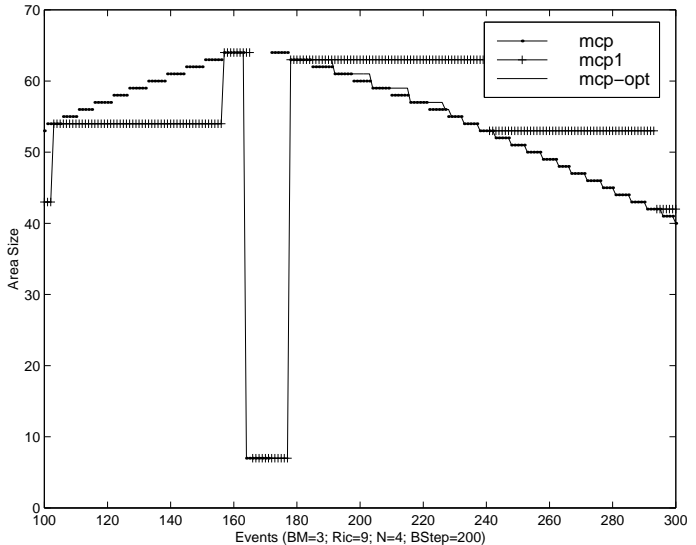

(b)

Figure 9: Instantaneous Location Area Size

is multiplied by BStep (i.e. 200) ${ }^{7}$. As a result, and as expected from equation 11, the location area increases. At event 165, the location area size decreases from 65 to 8 . This is because the mobile host has finished its big move and enters into a micro mode. The speed getting smaller, the location area size decreases. Then after few events (at event 150), the location area size increases again drastically from 8 to 60 : the mobile host has one more time moved into a big move mode. From event 180 to event 360 , the mobile stays in a big move mode and its location area size fluctuates between 65 and 40 because its distance to its home agent varies as it is moving (see figure 5(a)). The mobile host then goes into a small move mode and its location area size decreases and so on...Figure 9(b) is a zoomed-version of figure 9(a) between events 100 and 300. This figure shows that $m c p$ adapts its location area size more often than the two other schemes. It actually adapts it as soon as the mobile host computes a new size. $m c p 1$ has to wait until the next location update. This is particularly noticeable after a big move. In fact, between events 160 and 180, it can be clearly seen that even though the mobile host goes into a small move mode, $m c p 1$ waits for awhile before adjusting its location area size. mcp_opt location area size adaptiveness is faster than $m c p 1$ but slower than mcp. mcp_opt adjusts its location area size only when this is cost effective. For example, it adapts as quickly as $m c p$ when it goes from a big move to a small move mode because the location area size decreases significantly and the paging cost with an outdated location area size would then be too expensive. However it adapts as slowly as $m c p 1$ when going from a small to a big move mode to avoid the location updating cost.

\footnotetext{
${ }^{7}$ As explained in section 5, both a big move and a small move occur during one time unit. Therefore a mobile host moves BStep faster during a big move than during a small move.
} 


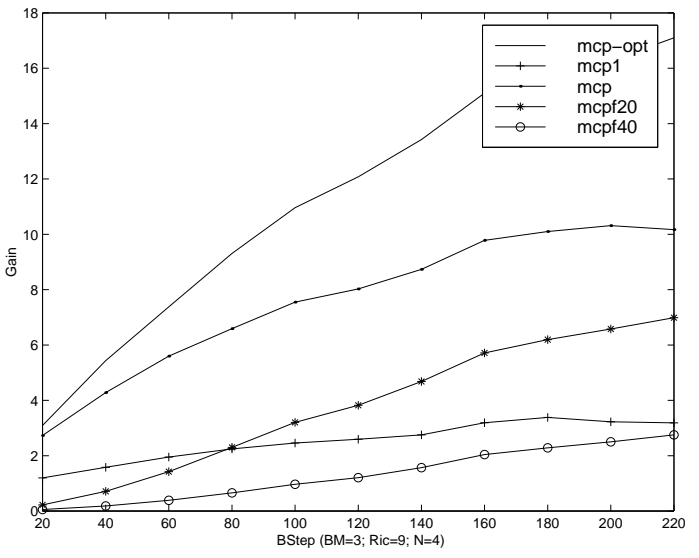

(a) Gain as a function of the big move size

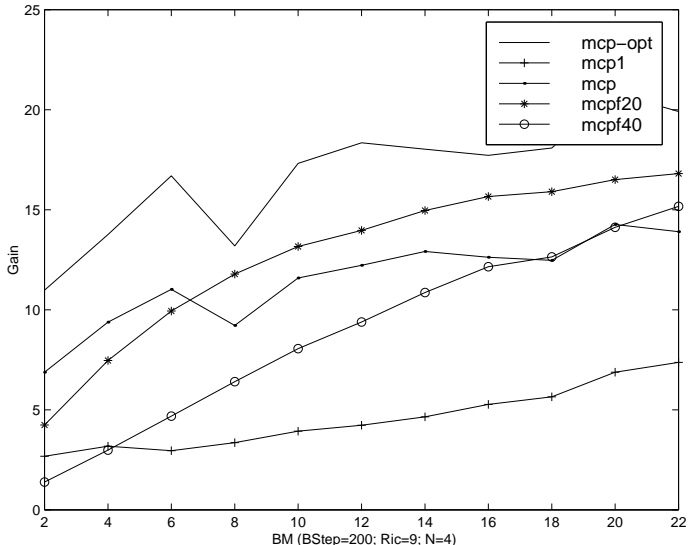

(b) Gain as a function of the number of big moves

Figure 10: Signaling Gain

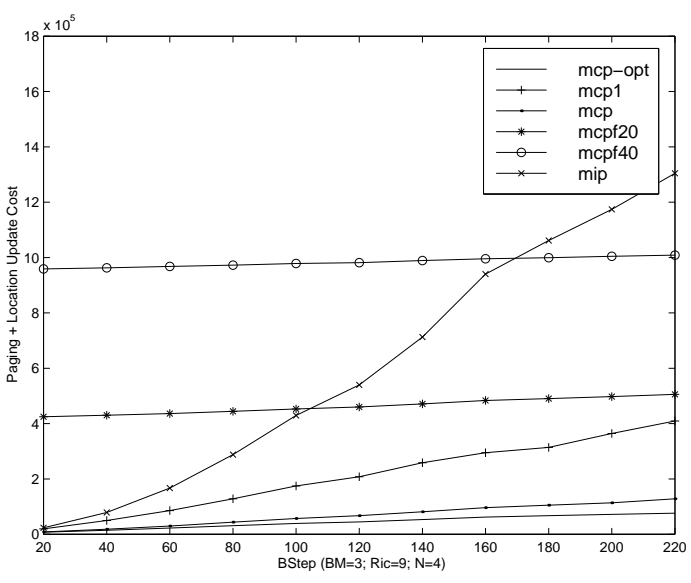

(a) Cost as a function of the big move size

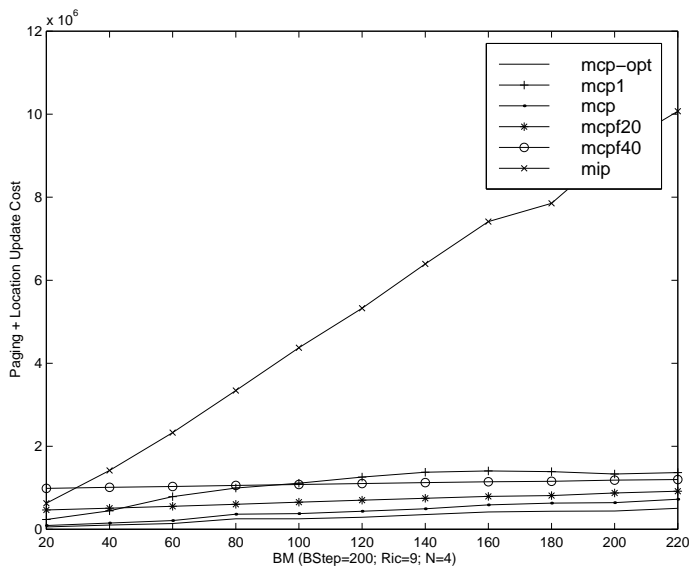

(b) Cost as a function of the number of big moves

Figure 11: Signaling Cost

Figure 10 shows the gain of $m c p, m c p 1, m c p \_o p t$ as a function of respectively the big move step size (BStep) and the number of big moves $(B M)$. It shows that $m c p_{-} o p t$ always out-performs the 
others schemes and that all gains increase as the step size and the number of big moves increase. These results are explained in Figure 11 which compares the signaling cost of each proposed scheme with the signaling cost of Mobile IP. The cost of Mobile IP increases much faster than the cost of $m c p f, m c p, m c p 1$ and $m c p \_o p t$. Consequently, the achieved gains increase with the size of the big moves and the number of big moves.

\section{Hierarchical Mobility Management Performance}

We then compare the performance of these different paging schemes with the performance of $H M I P$, a hierarchical mobile IP scheme [5]. With HMIP, mobility management is divided into two levels: the macro and the micro levels. When a mobile host moves from one domain to another one, it gets a new global CoA (Care of Address) which is valid as long as it stays within the new domain and registers it with its home agent using Mobile IP. When the mobile host moves within a domain, it gets a local care-of address and registers it with the domain gateway (the home agent is unaware of the MH's local moves) which keeps a binding between the MH's home address, its global address and its local address. When a packet addressed to the mobile host reaches its home network, it is intercepted by the mobile host's home agent and forwarded to its global CoA [11]. When the packet reaches the gateway, it is encapsulated to the mobile host local CoA.

When simulating HMIP, we divided our grid of base stations into domains. A domain is defined as a square of $k \times k$ cells. The gateway of a domain is located at its center. If a host moves within a domain it registers with the local gateway. If it moves into a new domain, it registers its new domain with its home agent and it registers its home address with the new gateway.

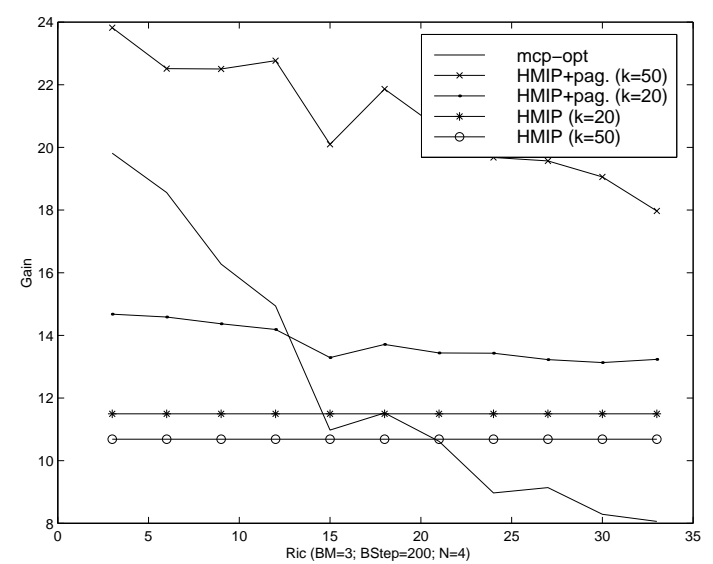

(a) Step Size $=200$

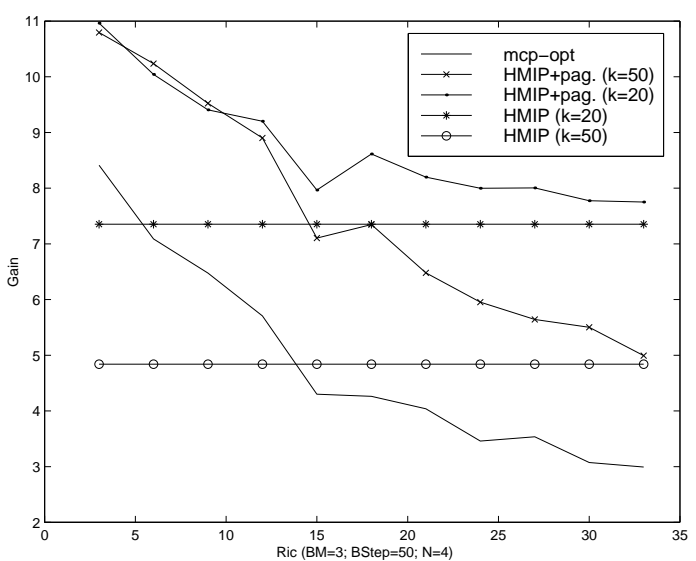

(b) Step Size $=50$

Figure 12: Signaling Gain 
Figure 12 presents the results of our simulations for $k$ equals to 20 and 50. These results show that $m c p_{-}$opt performs better than HMIP for small number of incoming calls par period (day). However HMIP performs better than mcp_opt for large number of incoming calls per period (i.e. larger than 15). This shows the limitation of paging mechanisms for large numbers of incoming calls.

\section{Combining dynamic paging with a hierarchical mobility management scheme}

In this section, we present the result of the integration of an individual adaptive paging scheme, namely $m c p_{-} o p t$, within HMIP. By combining paging and hierarchical mobility management, we are hopping to achieve good performance for small and large $R_{I C}$. With this paging-extended HMIP scheme, a mobile host computes its optimal location area as explained in Section 4. However instead of registering with its home agent as in section 3, it registers with it local gateway as long as it is moving within the same domain. A mobile host registers with its home agent only when it moves into a new domain. When the local gateway receives a packet for a mobile host of its domain it sends it to the MH's PA which pages the mobile host. In response to this paging request, the mobile host registers its current location with the gateway and forthcoming packets are directly forwarded to it.

The results achieved with domain sizes of 20 and 50 are displayed in Figure 12 (the pagingextended HMIP scheme is referred as HMIP + pag.). This figure shows that the gain of our paging-extended hierarchical scheme is significantly better (up to two times better) than the gain achieved by HMIP for any value of $R_{I C}$. This gain in performance is explained by the fact that the MH does not report to its local gateway each time it moves but only when its receives a paging request. As a result, when the number of incoming call is small, significant gain is achieved. As $R_{I C}$ increases, the gains of the paging-extended HMIP and of HMIP converge. In fact, for large $R_{I C}$, the paging area size converges to 1 and both schemes become similar. Figure 12 also shows that the paging-extended HMIP performs significantly better than mcp_opt (up to two times better) because the cost of updating the location area is then much smaller.

\section{Future Work}

This paper shows that Mobile IP with some simple extensions, such as paging, can be made adapted to Cellular networks.

We believe that IP flexibility provides even further opportunities for improvements. In this paper, we considered that the incoming traffic was time-sensitive. As a result, the whole LA is paged whenever there is a new incoming call for a mobile host. For some non real-time data, successive concentric paging area could be used instead. This might further reduce the generated signaling load [2].

The results presented in this paper are preliminary. We used a simple simulator using simple models. The motivations for this choice was to get a good grasp of the problems and results. Future work should address the following issues:

- The network model that we used is quite simplistic. All cells have the same size and are uniformly distributed. More simulations are needed with irregular network models. 
- We considered hexagonal-shaped location areas. Shapes that take into account the mobile host mobility pattern may lead to better performance results [9].

- In our models, we used geographical distances instead of topological distances (i.e. number of nodes between two hosts of a network). The effect of this simplistic approximation needs to be studied in more details. Some simulations with a network simulator such as NS [1] are needed.

- We ignored caching behaviors. In fact a LA that receives a new call for a mobile host pages it even if the previous call has just terminated. The effect of caching need to be addressed.

- We did not consider the Mobile IP route-optimization [6] in this paper. Future work should study how this extension affects our proposal.

- We did not consider implementation issues. There are some of them that need to be addressed such as::

- we need to find an efficient way to page a location area. [13] proposes to use IP Multicast. In this proposal, a multicast address is assigned to each Base Station of a location area. A router pages a mobile host by sending a paging request to the LA multicast address. This might be a possible solution but more work is required especially for dynamic location areas.

- we assumed that the incoming call rate and mobility parameters (i.e., the speed and the distances to its home agent, mobility agent or PA) were known by the MH . This is not exactly true in the real world. These parameters need to be estimated. Future work should address the problem of how to design real-time estimators. The sensibility of our scheme to the estimation errors should also be studied.

\section{Related Work}

HAWAII [12] and Cellular IP [4] are two micro-mobility protocols that use a paging scheme. [12] and [4] present mechanisms to implement paging but do not describe how to configure location areas optimally. In contrast, and as in [14], we define an algorithm that finds the optimal location area of each mobile host without going into too much details of its implementation. We believe that our scheme can be deployed within HAWAII and Cellular IP and that some of the mechanisms used in these two micro-mobility protocols could be used in a paging-extended Mobile IP protocol. The rest of this section presents briefly the HAWAII and Cellular IP paging protocols and outlines the differences between our proposal and the dynamic paging scheme defined in [14].

HAWAII [12] uses a paging mechanism that has been designed to minimize signaling load and reduce battery power consumption. [13] describes the paging protocol that relies on IP Multicast. A domain is divided in paging areas. All the Base Stations of a paging area are part of a multicast group. [13] do not define any specific way of defining paging areas. Fixed PAs can be statically 
configured with different multicast group addresses. In order to support dynamic paging areas, Base Stations may have to join multicast groups in a dynamic fashion. The feasibility and efficiency of such solution need to be studied more deeply.

In Cellular IP [4], the network operator can group the cells into Paging Areas. An idle mobile host that moves into a new Paging Area must transmit a paging-update packet. Paging-update packets are routed from the Base Station to the Gateway using hop-by-hop routing. Selected nodes of the Cellular IP network are equipped with Paging Cache. These nodes monitor passing pagingupdate packets and update Paging Cache mappings to point toward the new Paging Area. When an IP packet arrives at a Cellular IP node, addressed to a mobile host for which no up-to-date Route Cache mapping is available, the Paging Cache is used to route the packet. If the node has no Paging Cache, it forwards the packet to all Down-link neighbors. A node that has Paging Cache but has no mapping in it for the destination mobile host discards the packet. On the path from the gateway to the mobile host there may be Cellular IP nodes with and without Paging Cache mixed. After the paging packet leaves the last node which has a Paging Cache it is effectively broadcast down-link by all nodes it passes. The set of cells that are reached by the paging packet forms a Paging Area. The number, size and population of Paging Areas in a Cellular IP network are determined by the topology of the network and the placement of Paging Caches. If a Base Station has a Paging Cache itself then it is alone in its Paging Area. If there are no Paging Caches in a Cellular IP Network, then the whole network is one Paging Area and paging becomes broadcasting.

In [14], a dynamic paging scheme that uses a individual adaptive location area per mobile host is defined. This proposal has been an inspiration for our work. However few differences exist between these two proposals: (1) [14] considers the radio links as the key resources and their scheme relies on this assumption. As a result, this proposal does not consider the cost of updating the location servers such as the home agent. Our scheme considers the whole network, i.e., the wired and wireless network. (2) We used hexagonal-shape cells and location areas in our network model. [14] uses squared-shaped cells and location areas. We believe that hexagonal-shaped cells and LA are more realistic. (3) In [14], a mobile host re-computes its optimal location area size only when it moves out of its current location area (this corresponds the scheme that we called $m c p 1$ in the simulations presented in section 5). In contrast, in our proposal, a mobile host computes its optimal location area size after each move and changes it if the cost of sending a binding update is lower than the cost that a paging with an outdated location area size might generate.

\section{Conclusion}

This paper proposes to extend Mobile IP with an individual adaptive paging scheme to reduce the generated signaling load in the network. In the proposed scheme, each mobile computes their optimal location area according to their mobility and incoming call parameters to reach the minimal signaling traffic. Simulations show that the proposed scheme saves significant bandwidth. More saving can even be achieved by combining our individual and adaptive paging protocol with a hierarchical scheme. 
We believe that simple extensions to Mobile IP can lead to significant performance gain and can make it adapted to Cellular networks. Micro-mobility protocols are actually not needed. IPbased cellular systems are motivated by the simplicity of the IP protocol stack and the low cost of IP routers. All extra complexity added to support mobile hosts might weaken this motivation. We argue that IP-based cellular systems should be kept simple with minimal support from the network.

\section{Acknowledgement}

The author thanks the members of the INRIA PLANETE group for their careful readings of this paper.

\section{References}

[1] UCB/LBNL/VINT Network Simulator - ns. In http://www-mash.CS.Berkeley.EDU/ns/, 1998.

[2] Timothy X. Brown and Seshadri Mohan. Mobility management for personal communications systems. IEEE Transactions on Vehicular Technology, 46(2):269-278, 1997.

[3] Ramon Caceres and Venkata N. Padmanabhan. Fast and Scalable Handoffs for Wireless Internetworks. In Proc. 2st Annual International Conference on Mobile Computing and Networking, 1996.

[4] A. Campbell, J. Gomez, C-Y. Wan, Z. Turanyi, and A. Valko. Cellular IP. Internet Draft, work in progress , draft-valko-cellularip-01.txt, 1999.

[5] Claude Castelluccia. A Hierarchical Mobile IPv6 Proposal. In INRIA Technical Report 226, 1998.

[6] David B. Johnson and Charles Perkins. Route Optimization in Mobile IP. Internet Draft, work in progress, 1997.

[7] David B. Johnson and Charles E. Perkins. Mobility Support in IPv6. Internet Draft, work in progress, 1998.

[8] Mohamed Khalil, Haseeb Akhtar, Emad Qaddoura, Charles Perkins, and Alberto Cerpa. Buffer Management for Mobile IP. Internet Draft, work in progress, 1998.

[9] Z. Lei and C. Rose. Wireless Subscriber Mobility management using Adaptive Individual Location Areas for PCS systems. In Proc. IEEE International Conf. on Communications (ICC'98), pages 1390-1394, Atlanta, Georgia, 1998.

[10] M. Mouly and M.B. Pautet. The GSM System for Mobile Communication. ISBN: 2-95071900-7, 1992.

[11] C. Perkins. IP Mobility Support. RFC 2002-bis, 1999.

$\mathrm{RT} \mathrm{n}^{\circ} 0236$ 
[12] Ramachandran Ramjee, Thomas La Porta, Sandy Thuel, and Kannan Varadhan. IP Micromobility Support Using HAWAII. In Internet draft, work in progress, 1999.

[13] Ramachandran Ramjee, Thomas La Porta, Sandy Thuel, and Kannan Varadhan. Paging Support for IP Micro-mobility Using HAWAII. In Internet draft, work in progress, 1999.

[14] H. Xie, S.Tabbane, and D.J. Goodman. Dynamic Location Area Management and Performance Analysis. In IEEE VTC, pages 536-539, Secausus, New Jersey, 1993. 


\section{Table of Contents}

1 Introduction $\quad 3$

2 Introduction $\quad 3$

3 Extending Mobile IP with individual adaptive paging 4

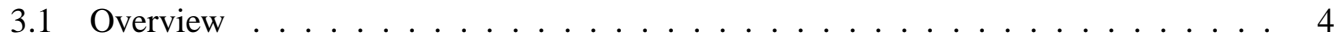

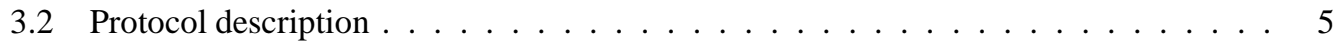

4 Location area optimal size computation $\quad 8$

4.1 Signaling Cost Function $\ldots \ldots \ldots \ldots \ldots \ldots$

4.2 Optimal Location Area Size Derivation $\ldots \ldots \ldots$

5 Performance Analysis $\quad 10$

5.1 Simulation Model . . . . . . . . . . . . . . . . . . . . . . . . . . . . . . 10

5.2 Analysis . . . . . . . . . . . . . . . . . . . . . . . 12

6 Future Work 18

7 Related Work 19

8 Conclusion $\quad 20$

9 Acknowledgement $\quad 21$

$\mathrm{RT} \mathrm{n}^{\circ} 0236$ 
Unit`e de recherche INRIA Lorraine, Technop^ole de Nancy-Brabois, Campus scientifi que, 615 rue du Jardin Botanique, BP 101, 54600 VILLERS LÈS NANCY

Unit'e de recherche INRIA Rennes, Irisa, Campus universitaire de Beaulieu, 35042 RENNES Cedex

Unit'e de recherche INRIA Rh^one-Alpes, 655, avenue de l'Europe, 38330 MONTBONNOT ST MARTIN

Unit'e de recherche INRIA Rocquencourt, Domaine de Voluceau, Rocquencourt, BP 105, 78153 LE CHESNAY Cedex

Unit'e de recherche INRIA Sophia-Antipolis, 2004 route des Lucioles, BP 93, 06902 SOPHIA-ANTIPOLIS Cedex

Éditeur

INRIA, Domaine de Voluceau, Rocquencourt, BP 105, 78153 LE CHESNAY Cedex (France)

http://www.inria.fr

ISSN 0249-6399 